\title{
Practical Byzantine Consensus for Internet-of-Things
}

This paper was downloaded from TechRxiv (https://www.techrxiv.org).

\section{LICENSE}

CC BY 4.0

SUBMISSION DATE / POSTED DATE

24-11-2021 / 07-12-2021

\section{CITATION}

Goyal, Himanshu; Saha, Sudipta (2021): Practical Byzantine Consensus for Internet-of-Things. TechRxiv. Preprint. https://doi.org/10.36227/techrxiv.17040188.v1

$\mathrm{DOI}$ 


\section{Practical Byzantine Consensus for Internet-of-Things}

\author{
Himanshu Goyal \\ IIT Bhubaneswar \\ hg11@iitbbs.ac.in
}

\author{
Sudipta Saha \\ IIT Bhubaneswar \\ sudipta@iitbbs.ac.in
}

\begin{abstract}
Use of IoT/WSN assisted smart-systems in the current age is making our living much more easier. However, components of such systems bear a high chance of getting compromised which may result in a substantial damage or loss. Use of fault tolerant consensus protocols provides a way towards solving this problem. Existing solutions for IoT/WSN systems mostly assume simple non-Byzantine node failures which is not enough to solve the problem. To combat the presence of smart devices with malicious intention, Byzantine fault tolerance support is highly essential in building trustworthy decentralised system. Byzantine fault tolerance has not been addressed much in the context of IoT/WSN because of its inherent requirement of extensive data sharing among the nodes. In this work, we approach to bring a solution to the problem using synchronous communication. In particular, we recast the well-known Practical Byzantine Fault Tolerant (PBFT) consensus strategy to an efficient form that is suitable for use in IoT/WSN systems. We demonstrate that our proposed design can work upto $80 \%$ faster and consume upto $82 \%$ lesser energy compared to a naive implementation of the strategy in publicly available IoT/WSN testbed having 45 nodes.
\end{abstract}

Index Terms-Byzantine Fault Tolerance, Synchronous Concurrent transmissions, Wireless Sensor Networks, Internet of Things (IoT).

\section{INTRODUCTION}

Internet-of-Things (IoT) and Wireless Sensor Networks (WSN) technologies play a crucial role in building various smart-systems, e.g., automated surveillance-systems [1], structure monitoring systems [2], smart-grid systems [3] and many others. Human civilization is tending to depend more on such smart-systems in near future. These systems are composed of large number of smart IoT-devices. Unfortunately, any such device is susceptible to various unexpected issues, e.g., software errors, failures or even attacks. Since these systems run through a close coordination among all it's components to achieve their target, such behavior can significantly impact on the overall system integrity. For instance, while monitoring a critical structure, a compromised IoT-device may wrongly report the status of the structure. Similarly, in a flock of UAVs with some mission, such issues may cause some of the UAVs to deviate from the target which in turn may lead to an overall failure and hence, incur a significant loss.

Fault tolerant consensus protocols play a vital role in establishing trustworthiness of a system in spite of the chances of node failures. Node failures can be either non-Byzantine or Byzantine. Consensus protocols to handle non-Byzantine failure assume a weak failure model, e.g., simple fail-stop or node crash and are mostly used in systems within a controlled environment, e.g., data center, where it can be controlled by authenticated entities. In contrast, consensus protocols capable of handling Byzantine failures [4], entail more comprehensive failure models where nodes can be compromised and have malicious intent behind participation. It is applicable for both open and decentralized systems, e.g., BlockChain $[5,6]$.

In the context of IoT/WSN based smart-systems, there are many works which achieve fast consensus, e.g., Chaos[7], A2[8], but without considering any failure possibilities. The work [9] realizes the well-known consensus protocol Paxos using Chaos as a base. But, the work considers only nonByzantine faults. However, support for Byzantine failures to build a trustworthy IoT system is inevitable in near future. However, there is no efficient work so far which attempts to achieve the same for IoT/WSN. In this work, we take an endeavor to support IoT/WSN systems with Byzantine fault tolerance. In particular, in this work we recast the well-known Practical Byzantine Fault Tolerant (PBFT) [10] consensus strategy to an efficient form suitable for IoT/WSN. Theoretically, an implementation of PBFT needs repeated data sharing among the nodes which would cause a lot of performance issue in low-power resource constrained systems like IoT/WSN in terms of latency and energy-consumption. In this work, we appropriately adopt synchronous communication based data sharing strategies to address this issue. The contributions from the work is summarized as follows.

- The well-known Byzantine fault tolerant consensus protocol PBFT has been recast to fit with synchronous communication framework for use in IoT/WSN systems.

- Optimisations over PBFT has been proposed in order to reduce overall completion time and energy consumption.

- The proposed design has been implemented in Contiki operating system for TelosB devices.

- The performance of the protocol has been evaluated in both simulator as well as publicly available IoT/WSN testbeds.

The rest of the paper is organized as follows. Section II provides a glance at some related works. Section III provides the necessary background regarding Byzantine fault tolerance as well as existing synchronous transmission based strategies along with the challenges in porting PBFT to WSN/IoT systems. Section IV provides the design details. Section V 
demonstrates an in-depth evaluation of the proposed strategy along with comparison with a naive implementation of the strategy. Section VI concludes the work along with some future directions.

\section{RELATED WORK}

Several solutions to the consensus problem with nonByzantine failures have been proposed in the literature [11, 12, 13, 14]. Paxos [12] is one of the earliest and well-known non-Byzantine consensus protocol which was later expanded to the protocol Raft [14]. Both Raft and Paxos have been extensively used in many permission based environments, e.g., Google's globally distributed File System [15], BlockChain enabled Hyperledger Fabric [16] etc. There are solutions to achieve consensus under Byzantine failures, e.g., Proof-ofwork (PoW) which is adopted in the BlockChain technology used in BitCoin [5], Proof-of-Stake (PoS) used in PPCoin[17]. They solve the problem using a simple logic. In PoW, in each round, a node is picked up with a probability proportional to its ability to solve computational puzzles, whereas in PoS, its selection in each round is decided based on either its stake or computation power.

All these works are mostly adopted for the distributed environment comprised of traditional resourceful desktop/server machines. An IoT system, in contrast, is mostly composed of low-power resource-constrained devices. Wireless-Paxos [9] is the first work to achieve fault tolerant consensus suitable for such resource-constrained system efficiently. However, it focuses only on handling crash-faults by leveraging the aggregation properties of another protocol Chaos [7], and fails to handle Byzantine failures. There are works [18, 19, 20, 21] that try to provide BlockChain-based services for IoT devices. However, to avoid carrying out in-network Byzantine fault tolerant consensus, they consider BlockChain network and IoT system to be different and interacting with each other through cloud. Initially, the devices submit their transaction requests to the local network which are then forwarded to the cloud based BlockChain network, e.g., Hyperledger Fabric [16] or Ethereum [22] and the result is sent back to the IoT-system. However, such split architecture induces a significant delay and potential issues under higher demand.

In contrast to these approaches, in the current work we demonstrate how Byzantine fault-tolerant agreement can be achieved through in-network processing, and thereby reducing the latency and improving the overall system performance.

\section{BACKGROUND}

We use PBFT as the primary means of achieving Byzantine failure resilient consensus protocol for IoT/WSN-systems. Miguel Castro and Barbara Liskov, in their pioneering work [10] demonstrated how to achieve consensus in a systematic way in a distributed system, in general, with Byzantine failures. We follow the same model here as described below.

A quorum is defined as the minimum number of nodes to pass a decision in an assembly. In PBFT, a node, in order to achieve consensus considering various types of failures, needs to reach a quorum. The minimum number of nodes necessary to be present in a system to enable every node to reach a quorum in spite of the presence of at most $f$ nonByzantine failures is $2 f+1$ [10]. Conversely, under nonByzantine failures, for $N$ number of nodes the quorum size is $\frac{N}{2}+1$. Under Byzantine failures, in contrast, quorum size is $\frac{2 N}{3}+1$ [23]. A node in the system requesting for consensus is referred to as a client node. A designated primary node is supposed to start the actual series of operations to reach consensus. In IoT/WSN system the sink node itself can play the role of the client as well as primary node based on demand /situation. The protocol works in five phases as depicted in Figure 1(a) and briefed below.

1) Launch: A client node (c) initiates the process by sending a request message $R e q$ to the primary node.

2) PRE-PREPARE: The primary node, in order to achieve consensus, broadcasts Req to all the participating nodes.

3) PREPARE: All the nodes in the system, validate the correctness of the request in Req and broadcasts a Prepare message in the system conveying their opinion about it.

4) COMMIT: Once a node reaches the quorum for the current Req, it broadcasts a Commit message to all the other nodes to formally agree or disagree.

5) Execute and reply: When the COMMIT phase ends up with a consensus among all the nodes, the process completes with a globally agreed reply for the Req. However, when consensus is not achieved, view-change (VC) takes place as briefed below.

6) View-Change (VC): In PBFT, the three consecutive phases PRE-PREPARE, PREPARE and COMMIT together are considered to be a view formed under the leadership of the current primary node. If COMMIT fails to achieve consensus, first, a new primary node is decided, and next the new node initiates the whole process starting from PRE-PREPARE phase. This is termed as View-Change. With $f$ number of traitors in the system, in the worst case maximum $f+1$, view formation may be needed to achieve a consensus.

A consensus protocol to handle only non-Byzantine failures needs mainly two phases involving communication among the nodes, e.g., first primary node needs to disseminate the request message to all the other nodes in the network and subsequently collect the replies containing the opinion of all the nodes. However, PBFT as shown above has three defined phases PRE-PREPARE, PREPARE, and COMMIT where massive communication among the nodes is required. In particular, all these three phases involve data sharing among all the nodes so that each node can reach the quorum. The message exchanges in PRE-PREPARE are of $\mathcal{O}(n)$ while the same in both PREPARE and COMMIT phases is $\mathcal{O}\left(n^{2}\right)$. Moreover, the change of view may also take place several times. Therefore, execution of a naive implementation of PBFT in resource constrained IoT/WSN system with a general communication framework would incur substantial cost in terms of message passing and result in high energy consumption and latency requirement. For an efficient realization of PBFT, in this 


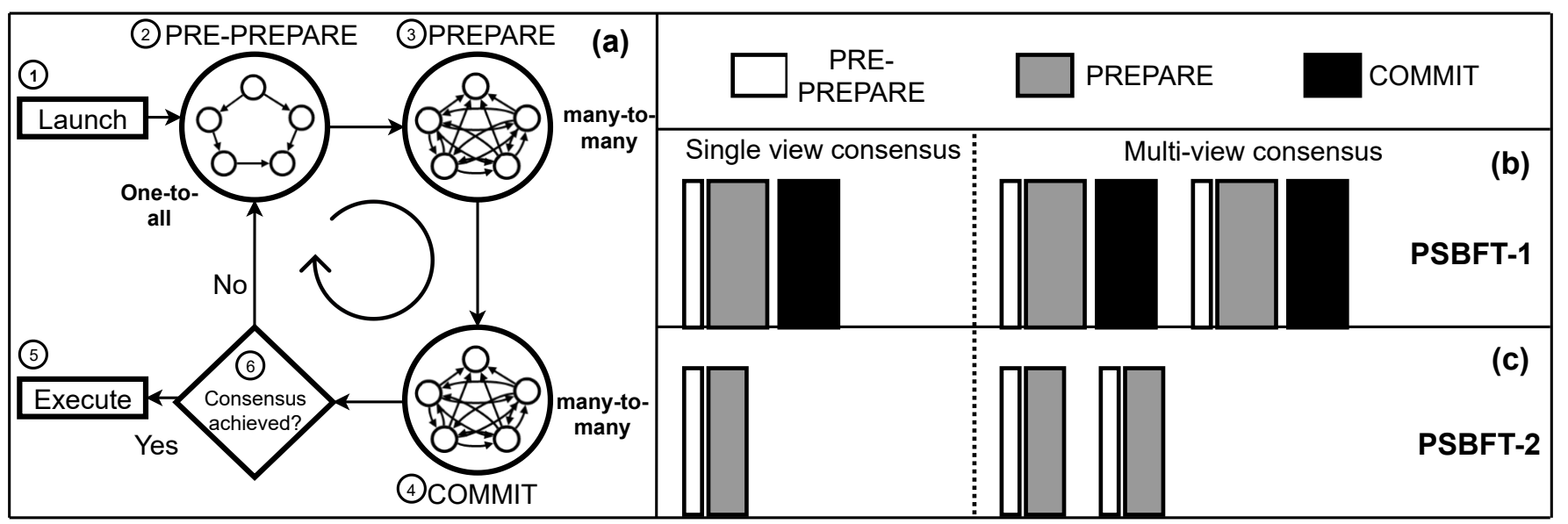

Fig. 1. Execution of PBFT. Part (a) shows the flow-diagram of a standard PBFT execution. Part (b) and (c) show the time-diagram of PBFT without optimizations and with optimizations, respectively.

work we make use of synchronous communication. A brief description about synchronous communication and the relevant protocols used in this work are provided below.

\section{A. Synchronous communication}

IoT/WSN systems mostly use low-power wireless communication. Under traditional asynchronous communication in broadcast-driven wireless medium, due to uncoordinated transmissions, the chance of collision rises quite fast when the nodes transmit more often. As a result, protocols like flooding, or many-to-many interaction incur a huge wastage of energy as well as time. In contrast, under synchronous/concurrent communication[24] the transmissions from different nodes are coordinated with the help of tight time-synchronization. This makes the transmissions overlap perfectly in time and trigger the physical layer phenomena called capture-effect which ensures that the packets are appropriately received in the respective receivers. There has been immense development in this field through many recent works such Glossy [25], Chaos [7], CodeCast [26], MiniCast [27], Mixer [28], LWB [29] etc. In the current work we use two protocols, Glossy and MiniCast for our purpose. These are briefed in the following.

Glossy: The work Glossy [25] for the first time demonstrated how synchronous transmission can be achieved through software based light-weight time synchronization in resource constrained IoT/WSN system. It also showed how synchronous transmission can be exploited for one-to-all flooding operation over a large multi-hop setting. The protocol works fully based on radio-events. The designated initiator node transmits the first packet which triggers the transmissions from the first hop neighbors of the initiator. Next, unlike asynchronous communication, all the first hop nodes transmit the same packet at the same time which results in capture effect in the receiver radios of the second hop nodes. The process continues this way until the flood reaches the full network. We use this protocol in our work as detailed in the next section.

MiniCast: In a single round of Glossy, only one initiator node becomes able to convey its data to all the other nodes in the network. The work MiniCast [27] demonstrates a
TDMA based strategy to merge multiple Glossy floods from different initiator nodes in a very compact way to enable efficient many-to-many/all-to-all data sharing. MiniCast works with the same principle as Glossy but allows the nodes to transmit the data in chain, using TDMA schedule. Unlike other works, MiniCast carries out the time-division multiplexing in the packet level to allow multiple Glossy floods to get interspersed with each other in an appropriate way reducing the latency and energy consumption in the nodes. In the current work we use MiniCast to address the major communication requirements of PBFT as detailed in the next section.

\section{DESIGN}

From the perspective of communication, the PREPRPPARE phase in PBFT would require a network-wide oneto-all data sharing initiated by the primary node. Similarly, both the PREPARE and the COMMIT phase would need allto-all data sharing in the network. In this work, we use one instance of Glossy for the PRE-PREPARE phase while two consecutive instances of MiniCast for the PREPARE and the COMMIT. This naive implementation of PBFT is referred to as Practical Synchronous Byzantine Fault Tolerant consensus protocol version-1, (PSBFT-1). Figure 1(b) shows the timing diagram of PSBFT-1. In the worst case, due to possible change of views, all the three phases may need to get repeated for a number of time. A new view is initiated by the PRE-PREPARE phase in case the COMMIT phase of the previous view fails to accomplish consensus among the nodes. Once consensus is accomplished, the next primary node does not start any further PRE-PREPARE phase and the nodes in the system turns off their radios after listening for a small and safe predetermined time period. While, this naive translation works as expected, we observe two potential scopes of improvement for enhancing the performance of the protocol as detailed below.

\section{A. Dynamic adjustment of NTX}

In PSBFT-1, in both PREPARE and COMMIT phases, allto-all data sharing is carried out in order for every node 

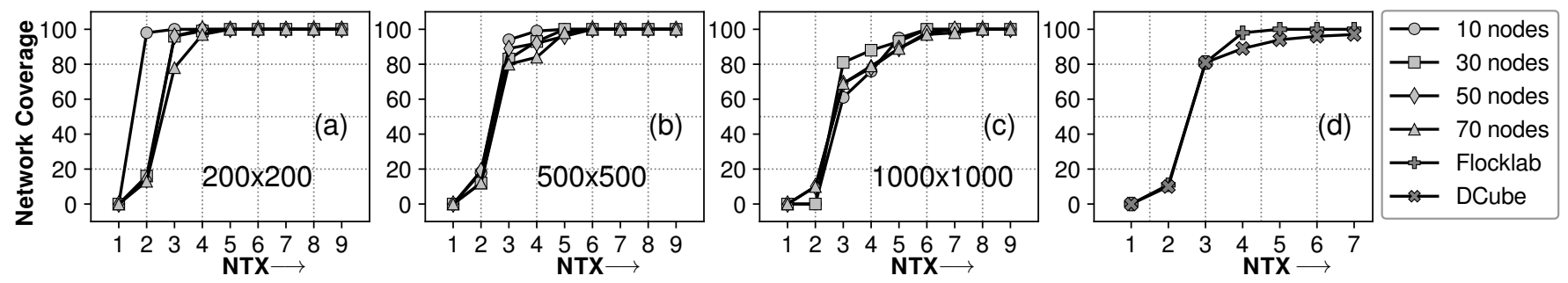

Fig. 2. Percentage of data received by the nodes with the variation of NTX.

to reach the quorum. However, strict all-to-all data sharing through any standard protocol is costly in terms of latency and energy consumption. As a general trend of the datasharing process in MiniCast, a good percentage of data is acquired by the nodes quickly through the initial receptions. But accumulation of new data items (from different nodes) slows down after that. Thus, true $100 \%$ data accumulation takes comparatively large time.

A quorum in a system is reached as soon as the required number of alike messages are received by the nodes. Thus, from the definition of the quorum size for Byzantine fault resilient consensus protocol, it can can be observed that strict all-to-all data sharing may not be always necessary. Rather, it depends on the effective number of traitors or faulty nodes in the system. In a system with $N$ nodes, although PBFT can support max $f$ traitors, where $N=3 f+1$, the actual number of traitors present in the system may be much lesser than $f$, say $k$. In this situation, the necessary size of the quorum is $2 k+1$. Thus, the underlying data sharing mechanism should ensure that each node receives at least $2 k+1$ alike messages. Assuming a random distribution of the traitors, by pigeonhole principle it can be shown that to reach the quorum, a node would need to receive at most $3 k+1$ messages/opinions. ${ }^{1}$ Thus, when there is a proper assessment of the number of traitors, there can be a substantial performance improvement over the PSBFT-1 by replacing strict all-to-all communication in both PREPARE and COMMIT phases by some-to-some or many-to-many communication.

Under synchronous transmission, both the protocols Glossy as well as MiniCast use a system parameter NTX to decide the number of times a node transmits and receives the data packets. In Glossy the transmission happens in the unit of a single packet while in MiniCast it happens in chain of packets where every source node is given a unique slot to transmit its data in that chain. Thus, the value of the NTX decides the degree of spread of the data from each of the source nodes. Conversely, this also makes the value of NTX capable of deciding the distinct number of nodes $(q)$ from which a node would receive the data. For $100 \%$ data reception the value of NTX is required to be fairly high. But when reception of data from only a fraction of the nodes is enough, NTX can be set to a much lower value. Thus, with knowledge

\footnotetext{
${ }^{1}$ Among $3 k+1$ replies, maximum $k$ replies can be different as they come from the $k$ traitors. Therefore, minimum $2 k+1$ replies must be same as they would come from the non-traitor nodes.
}

of the number of traitor $k$, the value of the NTX can be set in a way so that the quorum can be safely reached by each node. But the relationship between $q$ and NTX depends on the specific network structure. Hence, in this work we assume that in the bootstrapping phase, there will be a distinct process to empirically study this relationship for the specific network structure and later use this relationship for future use. Section V provides an empirical study to understand this relationship in various network settings. Once the sink node is provided with an idea regarding the traitors in the system, it first derives the value of NTX needed for the PREPARE and COMMIT phase and the same is subsequently disseminated through the Glossy round in the PRE-PREPARE phase.

\section{B. Redundancy of COMMIT phase}

Creating visible disturbances in a system [30, 31] always provides a chance for the administrator to get an alert about the presence of a traitor and help it to take exhaustive measure. Therefore, in general, a traitor acts rather in a silent/passive way. Hence, usually a traitor would not prefer to mess up with the communication system/framework. In an asynchronous or wire-connected system, in response to a query, a traitor node is free to reply differently to different nodes as well as change data while forwarding if possible. However, the protocols MiniCast and Glossy which we use to realize PBFT in this work, both are based on synchronous transmission where, in a given time slot, the transmitters are supposed to transmit the packets with same content. Thus, a traitor, in order to cooperate with the synchronous communication setting, usually would not prefer to reply differently to different nodes or change other nodes' data while being forwarded through them $[30,31]$. However, the traitors are free to pass wrong/imperfect opinion in their own packet. Synchronous communication, thus, naturally restricts the Byzantine capability of a traitor. Because of this restriction, in PSBFT-1, after completion of the PREPRAE phase, all the nodes acquire a consistent view which is quite good enough to decide whether consensus is achieved or view change is necessary. A separate COMMIT phase is, hence, redundant in our proposed setting.

We incorporate the two above mentioned optimizations over PSBFT-1 and refer to the modified version as PSBFT2. Figure 1(c) depicts the time-diagram of PSBFT-2 in comparison with PSBFT-1. The detailed evaluation study of the designed protocols are provided in the next section. 


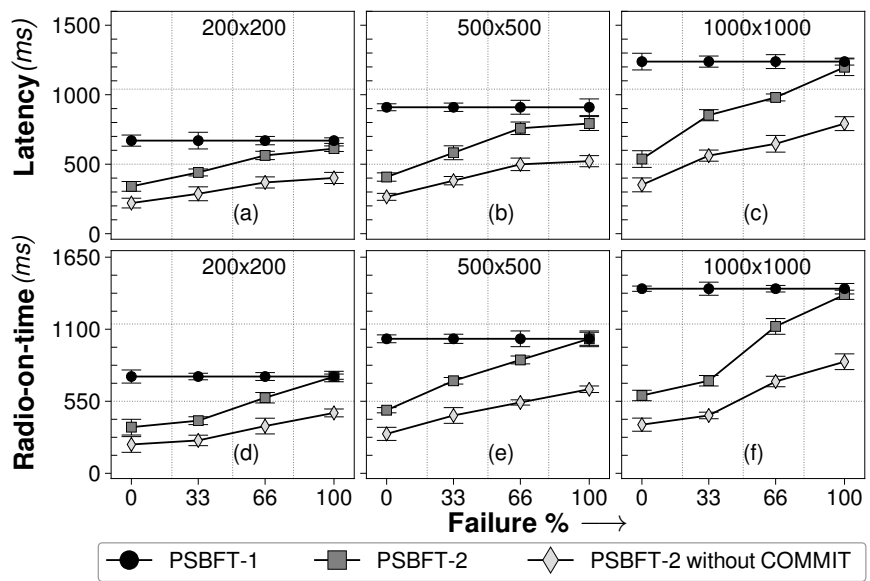

Fig. 3. Latency and Radio-on-time for 70 node simulation over various network diameter

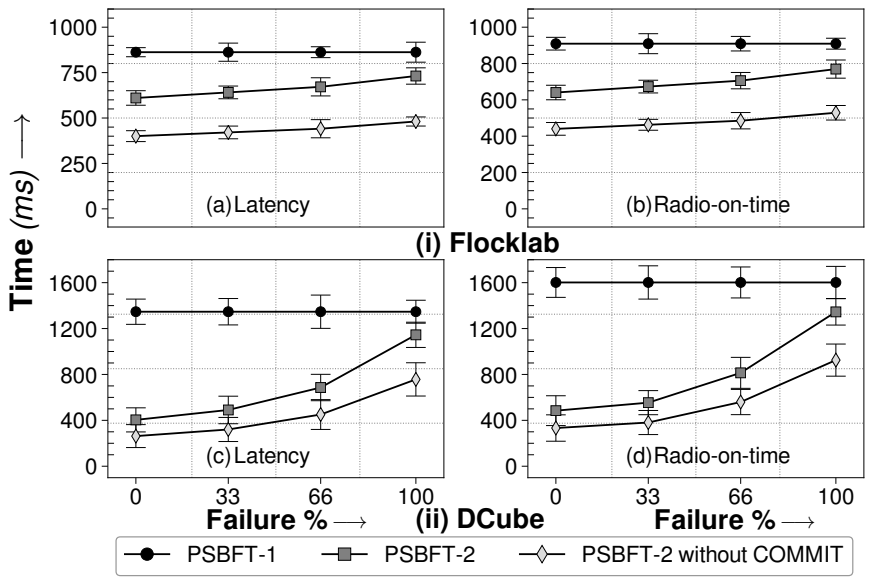

Fig. 4. Latency and Radio-on-time for Flocklab and DCube

\section{Evaluation}

We implement PSBFT-1 and PSBFT-2 in Contiki OS for TelosB motes. We simulate it using Cooja simulator. We also experiment with it in the publicly available IoT/WSN testbeds DCube [32] and Flocklab [33] composed of 45 and 24 TelosB motes, respectively. We first provide our experimental study over plane MiniCast [27] to determine the minimum value of NTX to be set to acquire opinions from sufficient number of distinct nodes to form a quorum of the necessary size. We run MiniCast in Cooja simulator over different network settings and check the data accumulation process. To quantify we use the metric Network Coverage calculated as the percentage of data received from the participating nodes in the network. For a given value of NTX, the network coverage $x$, signifies that all the nodes have received messages from $x \%$ of the nodes on average. Figure 2 highlights the variation of network coverage with NTX for different network configuration. Its observed from all these results that to achieve $100 \%$ network coverage we need to set the value of NTX above 7. But based on the number of nodes and the simulation area, the value of NTX needed to get upto $70 \%$ coverage is quite less. For a certain number of nodes, it increases with the area of deployment as visible in Figure 2 (a)-(c). The same trend is visible in testbed networks DCube and Flocklab as shown in the Figure 2 (d). In particular, the relationship depends on the specific network setting. To take the advantage of this fact, before carrying out experiments with PSBFT-2, we study this relationship in details for the target networks and prepare a table which we use while setting the value of NTX based on the requirement of the quorum size.

\section{A. Metrics}

To compare the performance of PSBFT-1 and PSBFT-2, we use the following two metrics.

Latency: It is the time taken for a process to achieve consensus. The value is calculated in each node and averaged over all the nodes and all the iterations.

Radio-on time: It is the total time necessary for a node to keep its radio-on to complete the execution of the protocol. The value is calculated in each node and averaged over all iterations and nodes.

\section{B. Results}

The performance of the PSBFT-1 and PSBFT-2 is compared under the presence of the Byzantine traitors under various network configurations through simulation. The number of traitors are varied from zero to the maximum number that can be supported by the algorithm (i.e., one third of the number of nodes). Figure 3 shows the comparison results for a 70 node simulation for different deployment area. Figure 4 shows the comparison results in FlockLab and DCube in terms of latency and radio-on time. In order to minutely understand the difference we also compare with an intermediate stage of PSBFT-2 with only NTX adjustment (i.e., without omission of COMMIT phase).

It can be observed that the performance of PSBFT-1 does not depend on the fraction of traitors in the system. PSBFT2 and its variant, performs the best when there is none or very less number of traitors which is quite obvious. PSBFT2 with COMMIT phase performs exactly same as PSBFT-1 when the fraction of the traitors is the maximum that can be supported. However, PSBFT-2 (with both the optimizations) performs always much better than PSBFT-1 even when there are many traitors in the system. In summary, in a 70-node network distributed over $1000 \times 1000 \mathrm{~m}^{2}$ simulation area, PSBFT-2 performs on average 52\% and upto $71 \%$ faster and consumes on average $58 \%$ and upto $73 \%$ lesser radio-on time in comparison to PSBFT-1 for a wide range of varying number of traitors randomly distributed over the network. The same experiments were repeated in DCube and FlockLab testbed networks. In Flocklab with 24 nodes, PSBFT- 2 was found to be performing on average $49 \%$ and upto $53 \%$ faster while consuming on average $52 \%$ and upto $55 \%$ lesser radio-on time in comparison to PSBFT-1. Similarly, in DCube with 45 nodes, PSBFT-2 performed on average $66 \%$ and upto $80 \%$ faster while consuming on average $68 \%$ and upto $82 \%$ lesser radio-on time in comparison to PSBFT-1. 


\section{CONCLUSION AND FUtURE DIRECTIONS}

Increasing dependence over the smart-system not only brings ease in living, but also introduces the possibility of unexpected problems because of the presence of smart-traitors in the systems. Existing consensus mechanisms for IoT/WSN mostly are capable of only managing non-Byzantine faults. To the best of our knowledge, the current work is the first attempt to efficiently realize Byzantine fault resilient consensus strategy for IoT/WSN systems. In general to build resilience against Byzantine faults in the system, the nodes needs to carry out extensive data sharing to exchange opinions and come to a conclusion. Therefore, traditional asynchronous transmission based communication mechanism for network communication does not fit well to solve this problem. In this work, we take the help of synchronous communication based efficient data sharing strategies and mould them appropriately to meet the requirements to efficiently realize the well known Byzantine fault tolerant consensus protocol PBFT for IoT/WSN systems.

Our evaluation results shows substantial improvement in the latency and energy-consumption in our proposed strategy to realize PBFT over its naive implementation. There are few interesting directions emerging from this work. To make the protocol fully self-sufficient and ready for practical use, an appropriate boot-strapping mechanism is necessary which can carry out a prior study to understand the appropriate value of NTX required to handle a given number of traitors. In the current work we carry out this step separately and use the information in the final protocol. Moreover, in this work we consider that the traitors, when more than one in number, are randomly distributed over the network. However, exact position of the traitors would have an effect on the protocol performance which needs to be studied in details. In the context of synchronous transmission where the nodes always transmit together and mostly its broadcast, effect of the traitors with the capability of data perturbation is also interesting to study. All these issues we plan to take up as our future endeavors in this direction.

\section{REFERENCES}

[1] V. A. Memos, K. E. Psannis, Y. Ishibashi, B.-G. Kim, and B. Gupta, "An efficient algorithm for media-based surveillance system (eamsus) in iot smart city framework," Future Generation Computer Systems.

[2] A. P. Plageras, K. E. Psannis, C. Stergiou, H. Wang, and B. Gupta, "Efficient iot-based sensor big data collection-processing and analysis in smart buildings," Future Generation Computer Systems.

[3] V. C. Gungor, B. Lu, and G. P. Hancke, "Opportunities and challenges of wireless sensor networks in smart grid," IEEE Transactions on Industrial Electronics.

[4] L. Lamport, R. Shostak, and M. Pease, "The byzantine generals problem," ACM Trans. Program. Lang. Syst., 1982.

[5] S. Nakamoto, "Bitcoin: A peer-to-peer electronic cash system."

[6] "Blockchain challenges and opportunities: A survey," Int. J. Web Grid Serv., 2018.

[7] O. Landsiedel, F. Ferrari, and M. Zimmerling, "Chaos: Versatile and efficient all-to-all data sharing and in-network processing at scale," in SenSys, 2013.

[8] B. Al Nahas, S. Duquennoy, and O. Landsiedel, "Network-wide consensus utilizing the capture effect in low-power wireless networks," in Proceedings of the 15th ACM Conference on Embedded Network Sensor Systems, pp. 1-14, 2017.

[9] V. Poirot, B. Al Nahas, and O. Landsiedel, "Paxos made wireless: Consensus in the air," in EWSN, 2019.
[10] M. Castro and B. Liskov, "Practical byzantine fault tolerance," in OSDI, 1999 (M. I. Seltzer and P. J. Leach, eds.), USENIX Association, 1999.

[11] L. Lamport, "The part-time parliament," ACM Trans. Comput. Syst., 1998.

[12] L. Lamport, "Paxos made simple," ACM SIGACT News (Distributed Computing Column) 32, 4 (Whole Number 121, December 2001), pp. 51-58, December 2001.

[13] L. Lamport, "Fast paxos," Distributed Computing, 2006.

[14] D. Ongaro and J. Ousterhout, "In search of an understandable consensus algorithm," in USENIX, 2014, 2014.

[15] J. C. Corbett, J. Dean, and E. et al., "Spanner: Google's globally distributed database," ACM Trans. Comput. Syst., 2013.

[16] E. Androulaki, A. Barger, and B. et al., "Hyperledger fabric: A distributed operating system for permissioned blockchains," in EuroSys, 2018.

[17] S. King and S. Nadal, "Ppcoin: Peer-to-peer crypto-currency with proofof-stake," 2012.

[18] O. Novo, "Scalable access management in iot using blockchain: A performance evaluation," IoT Journal.

[19] E. Schiller, E. Esati, S. R. Niya, and B. Stiller, "Blockchain on msp430 with ieee 802.15.4," in $L C N, 2020$

[20] C. Profentzas, M. Almgren, and O. Landsiedel, "Iotlogblock: Recording off-line transactions of low-power iot devices using a blockchain," in $L C N, 2019$

[21] C. Profentzas, M. Almgren, and O. Landsiedel, "Tinyevm: Off-chain smart contracts on low-power iot devices," in ICDCS, 2020.

[22] G. Wood et al., "Ethereum: A secure decentralised generalised transaction ledger," Ethereum project yellow paper, vol. 151, no. 2014, pp. 132, 2014.

[23] M. Van Steen and A. Tanenbaum, "Distributed systems principles and paradigms," Network, vol. 2, p. 28, 2002.

[24] M. Zimmerling, L. Mottola, and S. Santini, "Synchronous transmissions in low-power wireless: A survey of communication protocols and network services," ACM Comput. Surv., vol. 53, Dec. 2020.

[25] F. Ferrari, M. Zimmerling, L. Thiele, and O. Saukh, "Efficient network flooding and time synchronization with glossy," in IPSN, 2011.

[26] M. Mohammad and M. C. Chan, "Codecast: Supporting data driven innetwork processing for low-power wireless sensor networks," in IPSN, 2018.

[27] S. Saha, O. Landsiedel, and M. C. Chan, "Efficient many-to-many data sharing using synchronous transmission and tdma," in DCOSS, 2017.

[28] C. Herrmann, F. Mager, and M. Zimmerling, "Mixer: Efficient manyto-all broadcast in dynamic wireless mesh networks," in SenSys, 2018.

[29] F. Ferrari, M. Zimmerling, L. Mottola, and L. Thiele, "Low-power wireless bus," in SenSys, 2012.

[30] Z. He, K. Hewage, and T. Voigt, "Arpeggio: A penetration attack on glossy networks," in SECON, 2016.

[31] K. C. Hewage, S. Raza, and T. Voigt, "Protecting glossy-based wireless networks from packet injection attacks," in MASS, 2017.

[32] M. Schuß, C. A. Boano, M. Weber, and K. Römer, "A competition to push the dependability of low-power wireless protocols to the edge," in EWSN, 2017

[33] R. Trüb, R. Da Forno, L. Sigrist, L. Mühlebach, A. Biri, J. Beutel, and L. Thiele, "FlockLab 2: Multi-Modal Testing and Validation for Wireless IoT," in CPS-IoTBench 2020. 